\title{
Prevalence of amyloid deposition in mature healthy chickens in the flock that previously had outbreaks of vaccine-associated amyloidosis
}

\author{
Kanata IBI ${ }^{1)}$, Tomoaki MURAKAMI ${ }^{2) *}$, Wael Mohamed GODA ${ }^{1)}$, Naoki KOBAYASHI ${ }^{1)}$, Naotaka ISHIGURO ${ }^{3)}$ and \\ Tokuma YANAI ${ }^{1)}$ \\ ${ }^{1)}$ Laboratory of Veterinary Microbiology, Department of Veterinary Medicine, Gifu University, 1-1 Yanagido, Gifu-shi, Gifu 501-1193, Japan \\ 2) Laboratory of Veterinary Toxicology, Tokyo University of Agriculture and Technology, 3-5-8 Saiwai-cho, Fuchu-shi, Tokyo 183-8509, Japan \\ ${ }^{3)}$ Laboratory of Food and Environmental Hygiene, Department of Veterinary Medicine, Gifu University, 1-1 Yanagido, Gifu-shi, Gifu \\ 501-1193, Japan
}

(Received 14 January 2015/Accepted 2 May 2015/Published online in J-STAGE 18 May 2015)

ABSTRACT. Avian amyloid A (AA) amyloidosis is commonly observed in adult birds with chronic inflammation, such as that caused by bacterial infection. We previously described vaccine-associated AA amyloidosis in juvenile chickens. In this study, the prevalence of amyloid deposition was measured in mature healthy chickens that survived a previous outbreak of avian AA amyloidosis while they were juveniles. Herein, we analyzed the amyloid deposition in mature chickens and compared the prevalence of amyloid deposition with juvenile chickens obtained in our previous study (Murakami et al., 2013). We found that: 1) amyloid deposition in the liver was absent in mature chickens, while juvenile chickens had a rate of $24 \%$; 2) amyloid deposition in the spleen was observed in $36 \%$ of juvenile chickens and in $40 \%$ of mature chickens; 3 ) amyloid deposition in the pectoral muscle of mature chickens (43.75\%) was approximately half that of juvenile chickens $(88 \%)$. These results suggest that additional amyloid deposition in chickens previously exposed to AA amyloidosis may not worsen with age. Further, amyloid deposition in chickens may tend to regress when causative factors, such as vaccinations and/or chronic inflammation, are absent.

KEY WORDS: amyloidosis, chicken, regression, vaccination

doi: 10.1292/jvms.15-0029; J. Vet. Med. Sci. 77(10): 1241-1245, 2015

Amyloidosis is a class of diseases characterized by amyloid fibril deposition in local or systemic tissues [12]. Amyloid A (AA) amyloidosis is one of the most frequently encountered systemic amyloidoses. The precursor of amyloid A is serum amyloid A (SAA) protein, which is an acute-phase reactant synthesized in the liver in response to inflammatory signals [2]. Impaired SAA degradation and other factors can chronically elevate the SAA concentration, leading to the conversion of SAA into AA fibrils [14, 22]. However, the precise mechanism underlying amyloid fibril formation remains unclear.

Avian AA amyloidosis affects older birds secondary to inflammatory disorders, such as mycobacteriosis caused by Mycobacterium avium subspecies paratuberuculosis or ulcerative pododermatitis with bacterial infections, so-called "bumblefoot" $[1,3,9]$. Avian amyloidosis occurs most frequently in waterfowl, particularly Peking ducks [18, 21]. A similar condition found in chickens, known as amyloid arthropathy, is associated with Enterococcus faecalis infection [8, 20, 23]. Amyloid deposition was also occasionally observed in commercial fowls [6, 19]. Experimental AA

\footnotetext{
*Correspondence to: MuraKami, T., Laboratory of Veterinary Toxicology, Tokyo University of Agriculture and Technology, 3-5-8 Saiwai-cho, Fuchu-shi, Tokyo 183-8509, Japan.

e-mail: mrkmt@cc.tuat.ac.jp

(C)2015 The Japanese Society of Veterinary Science

This is an open-access article distributed under the terms of the Creative Commons Attribution Non-Commercial No Derivatives (by-nc-nd) License $<$ http://creativecommons.org/licenses/by-nc-nd/3.0/>.
}

amyloidosis in birds can be induced by repeated inflammatory stimulation, such as casein administration or vaccination with oil-emulsified bacterins [4, 10, 17].

In a previous study, outbreaks of fatal AA amyloidosis associated with vaccination in juvenile chickens were reported at a large poultry farm in Japan [13]. Notably, survived vaccinated chickens that were apparently healthy also showed amyloid deposition in several organs. In human and experimental murine model systems, AA deposition is known to decrease over time $[11,16]$. However, the duration of amyloid deposition in chickens is unclear. The aim of the present study was to assess the incidence of residual amyloid deposition by using mature chickens from a flock that had outbreaks of AA amyloidosis in the juvenile stage two years ago.

\section{MATERIALS AND METHODS}

History of animals: Fifty euthanized Julia-lite chickens were obtained from an egg farm. These chickens were two years old and previously reared at a large raising farm [13]. In the raising farm, chickens were introduced to the farm at about 70 days old, inoculated subcutaneously or intramuscularly with multiple vaccines in pectoral muscles at 90 days old. The multiple vaccines were composed of 1 dose of infectious coryza type $\mathrm{A}$ and $\mathrm{C}$ vaccine, 1 dose of $\mathrm{Myco}-$ plasma gallisepticum vaccine, 1 dose of Newcastle disease, infectious bronchitis and egg drop syndrome-1976 vaccine and one-half dose of Salmonella Enteritidis vaccine. During this maturation period, approximately $2 \%$ of the flock died 
due to severe vaccine-associated amyloidotic hepatitis, and 22 of $25(88 \%)$ randomly selected clinically healthy birds developed amyloid deposition in several organs [13]. The surviving chickens were transferred to an egg farm at about 130 days old and served as egg laying hens for 2 years. During production, the mortality rate and egg production of the chickens were normal. We were not able to survey the vaccination history during the egg laying period. After the production period, 50 randomly selected apparently healthy chickens were euthanized by farm producers and shipped to Gifu University for necropsy.

Macroscopic, histological and immunohistochemical examinations: All 50 chickens were necropsied. Liver, spleen, kidney, duodenum and pectoral muscle tissues were collected from each specimen, and samples of each organ were fixed in $15 \%$ neutral-buffered formalin. At the collection of pectoral muscle, the regions of inflammation were preferentially collected. The tissues were then embedded in paraffin, cut into $3-\mu \mathrm{m}$ sections and stained with hematoxylin and eosin (H\&E), congo red (CR), phosphotungstic acid-hematoxylin (PTAH), Masson's trichrome (MT), periodic acid-Schiff (PAS) or silver impregnation (SI). All amyloid depositions were detected using emerald-green birefringence identified in CR-stained sections under polarized light. The severity of amyloid deposition in each organ was graded visually in CR-stained sections as follows: (-) no amyloid deposition; $(+)$ mild amyloid deposition and $(++)$ moderate to severe amyloid deposition. Selection criteria for the severity of amyloid depositions are shown in Fig. 1. The grading was conducted by two pathologists (Ibi and Murakami).

Amyloid deposition was also detected by immunohistochemistry (IHC) using an Envision+ kit (Dako, Carpinteria, CA, U.S.A.) and an anti-chicken SAA1 mouse serum [15] as the primary antibody.

\section{RESULTS}

In several cases, caseating necrosis in the pectoral muscle at the vaccination site, a fatty liver, mild-to-moderate splenomegaly and peritonitis were observed on gross examination. Although the vaccination history during the laying period was unavailable, the site of caseating necrosis in the pectoral muscle was consistent with the vaccination site from two years before [13].

The most noticeable amyloid deposition was observed in the pectoral muscle in 21 of $48(48 \%)$ birds, followed by the spleen in 20 of $50(40 \%)$ birds (Table 1). The pectoral muscle showed necrosis, fibrous hardening and abscessation; amyloid was primarily deposited in the vascular walls and interstitial spaces within inflamed areas (Fig. 1a-1d). In the spleen, minimal to mild amyloid depositions were observed in the vascular walls. In the duodenum, minimal amyloid deposition was observed in only one chicken. Amyloid depositions were never found in the liver or kidney. The amyloid deposition in each organ was confirmed as amyloid A by IHC (Fig. 1c and 1d).

In addition to amyloid deposition, eosinophilic clots in the liver and eosinophilic fibriform accumulation in the spleen
Table 1. Severity of amyloid deposition in mature chickens

\begin{tabular}{crrrrc}
\hline $\begin{array}{c}\text { Degree of } \\
\text { changes }^{\text {a }}\end{array}$ & Liver & Spleen & Kidney & Duodenum & $\begin{array}{c}\text { Pectoral } \\
\text { muscle }\end{array}$ \\
\hline- & 50 & 30 & 50 & 49 & 27 \\
+ & 0 & 20 & 0 & 1 & 19 \\
++ & 0 & 0 & 0 & 0 & 2 \\
\hline Total & 50 & 50 & 50 & 50 & 48 \\
\hline
\end{tabular}

a) -, no; +, mild; ++, moderate to severe amyloid deposits.

were frequently observed as characteristic histological changes in the examined chickens. In the liver, the deposition of eosinophilic clots was identified in the interstitium, destroying the hepatic cell codes with mild fibrosis (Fig. 2a). These lesions appeared pale purple on PAS staining, pink to red on MT staining (Fig. 2b) and were negative on CR, PTAH and SI staining (Fig. 2c). By IHC, the eosinophilic clots were negative for anti-AA. In the spleen, perivascular fibriform accumulations were observed (Fig. 2d). These lesions appeared red on PTAH staining, blue on MT (Fig. 2e), dark purple on SI (Fig. 2f), light red on PAS staining and were negative on $\mathrm{CR}$ and against AA by IHC.

\section{DISCUSSION}

The comparison of the prevalence of amyloid deposition between juvenile chickens in the previous study [13] and mature chickens in this study showed that; 1) amyloid deposition in the liver was absent in mature chickens, while juvenile chickens had a rate of $24 \%$; 2) amyloid deposition in the spleen was observed in $36 \%$ of juvenile chickens and in $40 \%$ of mature chickens; and 3) amyloid deposition in the pectoral muscle of mature chickens $(43.75 \%)$ was approximately half that of juvenile chickens (88\%) (Fig. 3). These results suggest that the potential for amyloid deposition tends to not worsen with age.

In mice with experimentally induced AA amyloidosis, the amyloid depositions were cleared within 8 months, if there was no systemic inflammation [16]. Similarly, AA deposition in humans with rheumatic disease also gradually decreased during treatment with an interleukin-6 inhibitor, which inhibits SAA production [11]. AA fibrils are produced from SAA protein; therefore, when SAA production and inflammation are inhibited, amyloid fibrils are no longer produced. However, the mechanism underlying amyloid regression remains poorly understood. In the pectoral muscle of the chickens in this study, minute to moderate amyloid depositions were observed surrounding inflammatory foci, and minute amyloid deposition occurred in the spleen. These results suggest that amyloid regression does not occur systemically, but instead occurs locally within each organ. In the present study, we examined mature chickens two years after an outbreak of AA amyloidosis at a poultry farm. More detailed studies are needed that assess the laying period using field cases or establishment of an experimental model of amyloid regression to examine the cause and mechanism of amyloid regression. 

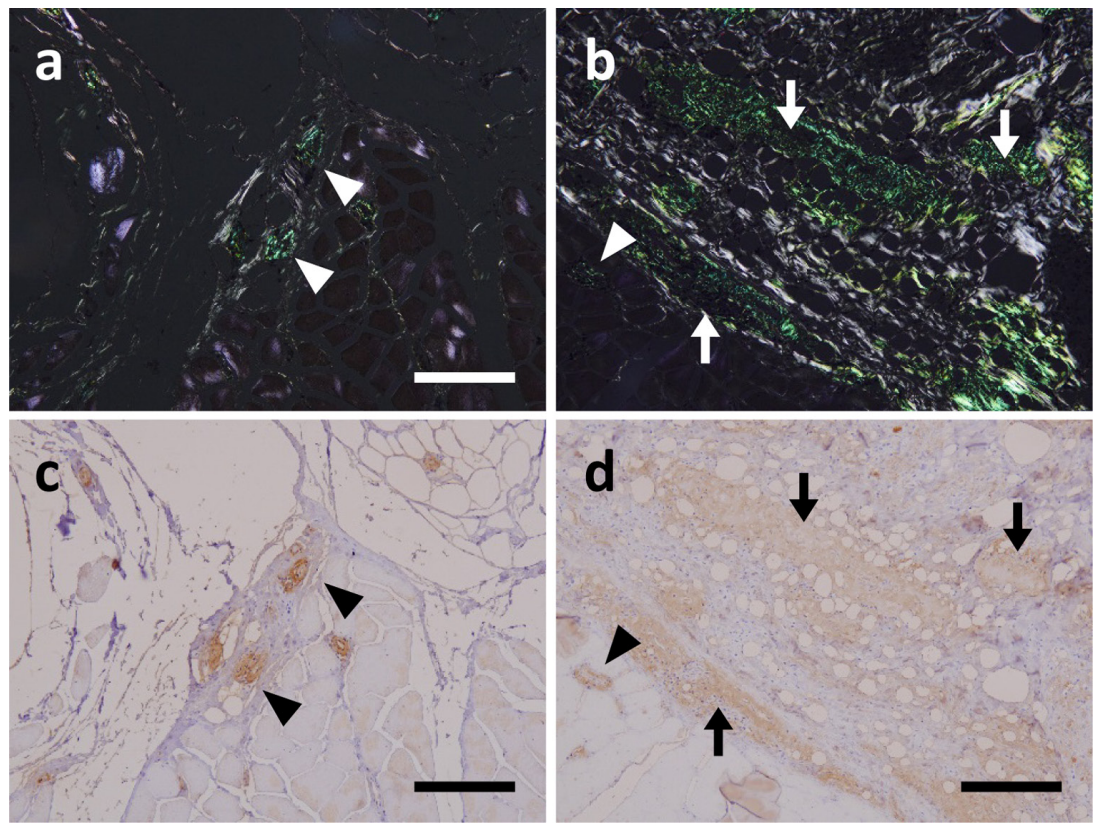

Fig. 1. Selection criteria for the severity of amyloid depositions. Serial section of pectoral muscles demonstrating the presence and degree of amyloid depositions. $(\mathrm{a}, \mathrm{b})$ Amyloid depositions were stained with Congo red and show green birefringence under polarized light. (c, d) Immunohistochemical labeling with an anti-chicken SAA mouse serum. The stained areas were identical to the Congo red positive areas. (a, c) Example of “+”. Amyloid depositions were observed just in vessel-walls. (b, d) Example of “++". Severe to moderate amyloid depositions were observed in interstitial spaces and vessel-walls. Scale bars=100 $\mu \mathrm{m}$.
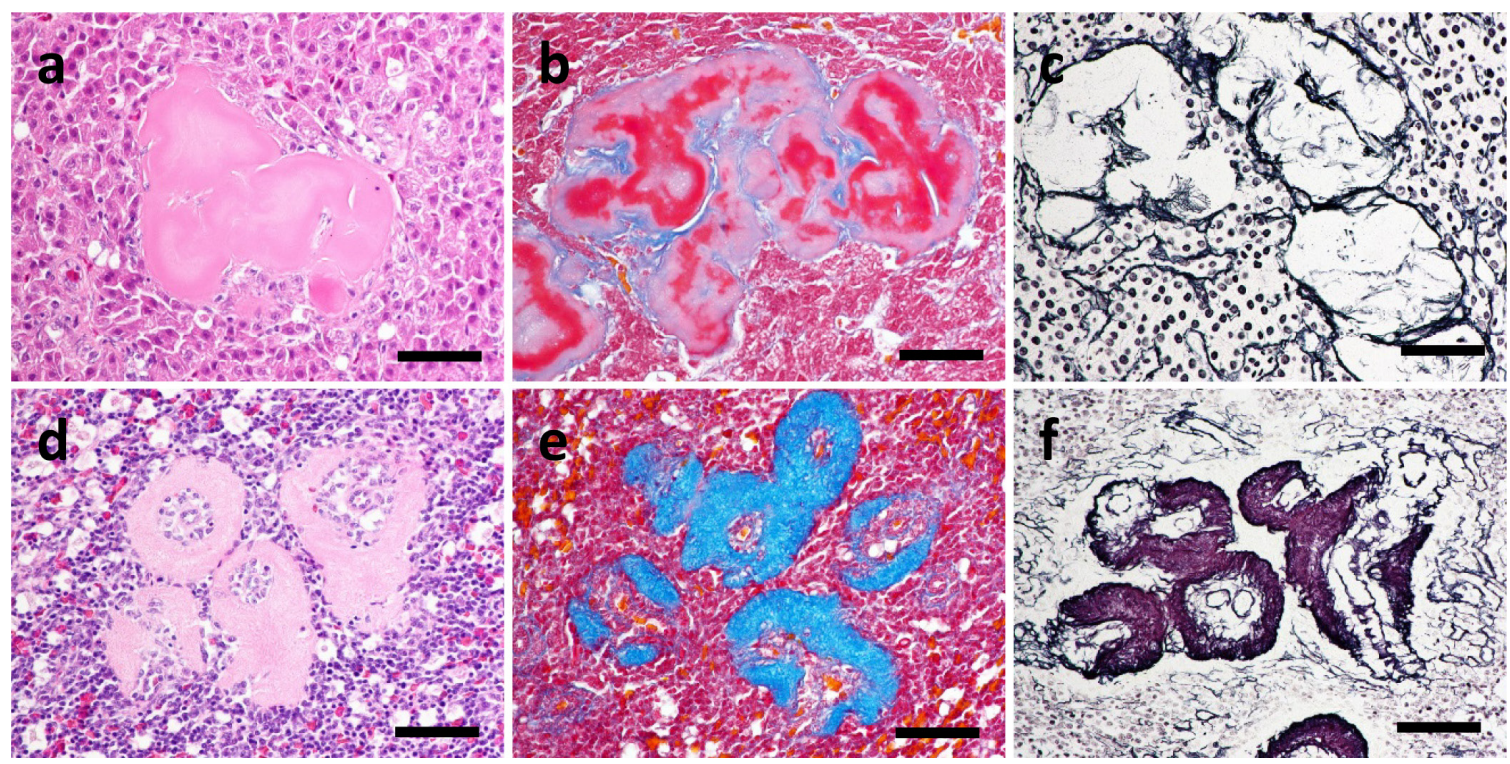

Fig. 2. Two characteristic histological lesions in the liver and spleen. (a-c) Eosinophilic clots (EC) in the liver. Scale bars=50 $\mu \mathrm{m}$. (a) Hepatic cell codes were destroyed by EC depositions (H\&E). (b) ECs appeared pale red to red with Masson's trichrome staining. The center of each EC stained more deeply than the peripheral areas. (c) ECs were negative with silver impregnation staining. (d-f) Fibriform accumulation (FA) in the spleen. Scale bars $=50 \mu \mathrm{m}$. (d) FAs were deposited in perivascular areas (H\&E). (e) FAs stained blue with Masson's trichrome staining. (f) FAs stained dark purple with silver impregnation staining.

Two characteristic histological lesions were observed in addition to amyloid deposition (Fig. 2). One was eosinophilic clots in the liver, which were suspected as hyaline degeneration from their histological form and amyloid-neg- ative-stainability. Previously, hemorrhagic inflammation and severe fibrin deposition were observed in the acute phase liver during the juvenile period [13]. Hyaline deposition may occur within hepatic regions emptied by fibrin retraction and 


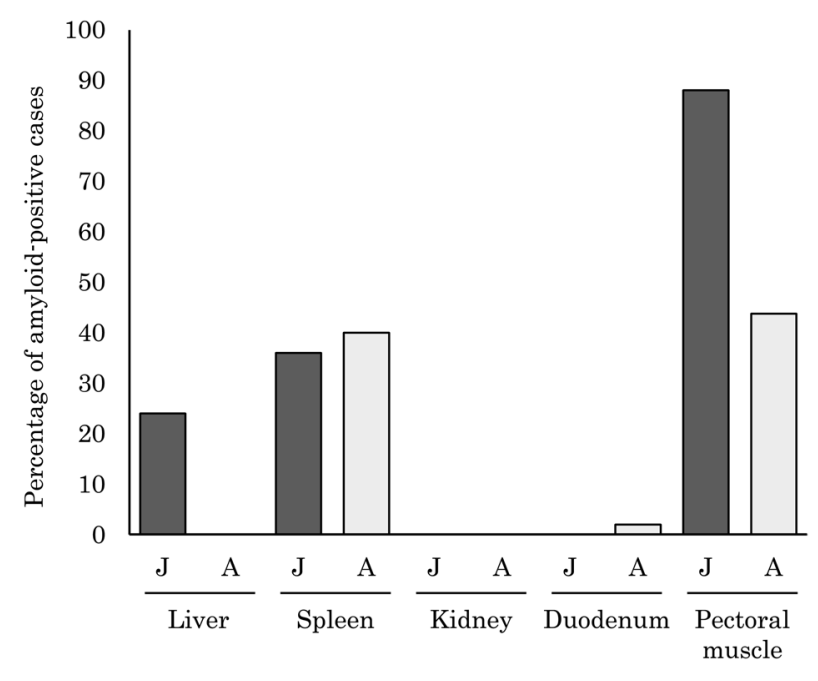

Fig. 3. Prevalence of amyloid deposition in juvenile chickens (J, dark bars) referred from the previous study [12] and mature chickens (A, pale bars) in this study.

coagulative necrosis. However, to confirm the relationship between hyaline and fibrin deposition, a long-term examination of laying chickens is required.

The other characteristic histological lesion was perivascular fibriform accumulation in the spleen, which was suspected as collagen fibers by special stainings [5]. These changes were not observed in the acute phase of vaccine-related AA amyloidosis during the juvenile stage [13]. As long as authors' experience, both characteristic changes in the liver and spleen are occasionally observed in mature avian species. Because of the similarity to amyloid deposition by H\&E staining, these two changes are likely misdiagnosed as amyloid deposition. Although the significance of these two lesions is still unknown, it is interesting that these changes were frequently observed in mature chickens from the flock that had outbreaks of vaccine-associated amyloidosis. To understand the pathogenesis of these amyloid-like lesions, it is preferable to track the stage of development over time.

In several mammalian species, renal amyloid deposition usually causes renal dysfunction [7]. Most of these animals quickly progress into nephrotic syndrome and have a poor outcome. However, specific clinical symptoms, such as nephrotic syndrome, are rare in avian species [9]. In the present study, renal amyloid deposition was not observed in any of the chickens examined. Furthermore, deceased chickens reared in the same flock did not develop renal amyloid deposition, despite the severe deposition in other organs. The cause of their death was thought to be not renal dysfunction, but rather hepatic disorder. The absence of renal amyloid deposition may accordingly prevent chickens from taking an acute course. Presumably, vaccine-associated chicken amyloidosis is a significant problem during acute phase inflammation, but amyloid depositions may not worsen in surviving birds, leading to good health through the entire laying period.
ACKNOWLEDGMENTS. This study was supported in part by a Grant-in-Aid (Emerging infection) for scientific research from the Ministry of Health, Labor, and Welfare of Japan and by a Grant-in-Aid for scientific research from the Institute of Medical Science, The University of Tokyo, and the Hokkaido University Center of Zoonosis Control.

\section{REFERENCES}

1. Cowan, D. F. 1968. Avian amyloidosis. I. General incidence in zoo birds. Pathol. Vet. 5: 51-58. [Medline]

2. Cray, C., Zaias, J. and Altman, N. H. 2009. Acute phase response in animals: a review. Comp. Med. 59: 517-526. [Medline]

3. Dias, J. L. and Montau, R. J. 1994. Staphylococcosis in captive exotic waterfowl. Avian Pathol. 23: 659-669. [Medline] [CrossRef]

4. Druet, R. L. and Janigan, D. T. 1966. Experimental amyloidosis. Amyloid induction with a soluble protein antigen in intact, bursectomized and thymectomized chickens. Am. J. Pathol. 49: 1103-1123. [Medline]

5. Farris, A. B., Adams, C. D., Brousaides, N., Della Pelle, P. A., Collins, A. B., Moradi, E., Smith, R. N., Grimm, P. C. and Colvin, R. B. 2011. Morphometric and visual evaluation of fibrosis in renal biopsies. J. Am. Soc. Nephrol. 22: 176-186. [Medline] [CrossRef]

6. Ishiguro, N., Murakami, T., Abdelazim, E. E. and Inoshima, Y. 2014. Surveillance of Amyloid Deposition and bacterial contamination in Chicken Liver from Meat Market. Jpn. Poult. Sci. 51: 104-107. [CrossRef]

7. Jakob, W. 1971. Spontaneous amyloidosis of mammals. Vet. Pathol. 8: 292-306. [Medline]

8. Landman, W. J., Gruys, E. and Dwars, R. M. 1994. A syndrome associated with growth depression and amyloid arthropathy in layers: a preliminary report. Avian Pathol. 23: 461-470. [Medline] [CrossRef]

9. Landman, W. J., Gruys, E. and Gielkens, A. L. 1998. Avian amyloidosis. Avian Pathol. 27: 437-449. [Medline] [CrossRef]

10. Ling, Y. 1992. Experimental production of amyloidosis in ducks. Avian Pathol. 21: 141-145. [Medline] [CrossRef]

11. Magro-Checa, C., Navas-Parejo Casado, A., Borrego-García, E., Raya-Álvarez, E., Rosales-Alexander, J. L., Salvatierra, J., Caballero-Morales, T. and Gómez-Morales, M. 2011. Successful use of tocilizumab in a patient with nephrotic syndrome due to a rapidly progressing AA amyloidosis secondary to latent tuberculosis. Amyloid 18: 235-239. [Medline] [CrossRef]

12. Merlini, G. and Bellotti, V. 2003. Molecular mechanisms of amyloidosis. N. Engl. J. Med. 349: 583-596. [Medline] [CrossRef]

13. Murakami, T., Inoshima, Y., Sakamoto, E., Fukushi, H., Sakai, H., Yanai, T. and Ishiguro, N. 2013. AA amyloidosis in vaccinated growing chickens. J. Comp. Pathol. 149: 291-297. [Medline] [CrossRef]

14. Murakami, T., Ishiguro, N. and Higuchi, K. 2014. Transmission of systemic AA amyloidosis in animals. Vet. Pathol. 51: 363-371. [Medline] [CrossRef]

15. Murakami, T., Muhammad, N., Inoshima, Y., Yanai, T., Goryo, M. and Ishiguro, N. 2013. Experimental induction and oral transmission of avian AA amyloidosis in vaccinated white hens. Amyloid 20: 80-85. [Medline] [CrossRef]

16. Nyström, S. N. and Westermark, G. T. 2012. AA-Amyloid is cleared by endogenous immunological mechanisms. Amyloid 19: 138-145. [Medline] [CrossRef]

17. Rampin, T., Sironi, G. and Gallazzi, D. 1989. [Episodes of 
amyloidosis in young hens after repeated use of antibacterial oil emulsion vaccines]. Dtsch. Tierarztl. Wochenschr. 96: 168-172, 170, 172. [Medline]

18. Rigdon, R. H. 1961. Amyloidosis: spontaneous occurrence in white Pekin ducks. Am. J. Pathol. 39: 369-378. [Medline]

19. Solomon, A., Richey, T., Murphy, C. L., Weiss, D. T., Wall, J. S., Westermark, G. T. and Westermark, P. 2007. Amyloidogenic potential of foie gras. Proc. Natl. Acad. Sci. U.S.A. 104: 10998-11001. [Medline] [CrossRef]

20. Steentjes, A., Veldman, K. T., Mevius, D. J. and Landman, W. J. 2002. Molecular epidemiology of unilateral amyloid arthropathy in broiler breeders associated with Enterococcus faecalis. Avian
Pathol. 31: 31-39. [Medline] [CrossRef]

21. Tanaka, S., Dan, C., Kawano, H., Omoto, M. and Ishihara, T. 2008. Pathological study on amyloidosis in Cygnus olor (mute swan) and other waterfowl. Med. Mol. Morphol. 41: 99-108. [Medline] [CrossRef]

22. Westermark, G. T. and Westermark, P. 2010. Prion-like aggregates: infectious agents in human disease. Trends Mol. Med. 16: 501-507. [Medline] [CrossRef]

23. Zekarias, B., Landman, W. J., Tooten, P. C. and Gruys, E. 2000. Leukocyte responses in two breeds of layer chicken that differ in susceptibility to induced amyloid arthropathy. Vet. Immunol. Immunopathol. 77: 55-69. [Medline] [CrossRef] 